Case Study

\title{
Surgical Evacuation Extradural Hematoma under Local Anesthesia
}

\author{
Peer Asad Aziz Qureshi ${ }^{1 *}$, Riaz. A. Raja ${ }^{2}$, Syed KhizrNaim ${ }^{3}$, Sanaullah pathan', Shahzor \\ Hassan $^{5}$ \& G.Murtaza Jamali ${ }^{6}$ \\ Registrar Neurosurgery Liaquat University of Medical Health Sciences \\ Corresponding Author: pirasadaziz@hotmail.com
}

\begin{abstract}
Extradural hematoma as a result of traumatic injury is a serious neurological emergency that required immediate intervention. The standard protocol is to identify the condition at earliest and provide quick surgical evacuation of the hematoma. The situation tends to be fatally deteriorating and marks a high mortality rate and worse health outcomes. We have presented a case of extradural hematoma in this study where the surgery had a delay due to the unavailability of general anesthesia. However, the falling Glasgow Coma Scale scores encouraged the surgeons to induce the surgical procedure with the employment of local anesthesia. The intervention turned out to be successful and produced the possibility of using local anesthesia as an alternative in case of critical emergency situations in order to save a life.
\end{abstract}

Keywords

Cranitomy, Local anesthesia, Extradural, traumatic brain injury, Extradural Hematoma

\section{Introduction}

Traumatic brain injury is a major public health concern that is foreseen to excel as a major reason of death and disability by $2020 .{ }^{1} \mathrm{~A}$ traumatic brain injury or TBI is caused as a result of collision, blow, shock, or penetrating wound to the head that disrupts the normal physiology of the brain. ${ }^{2}$ Centers for Disease Control and Prevention (CDC) has estimated that around 2.4 million visits in the emergency department and hospitals turned in with a traumatic brain injury. The incidence are presented either as a sole trauma or a combination of more than one injury. ${ }^{3}$ Approximately, $75 \%$ cases of the TBIs are of mild intensity and are termed as concussions. ${ }^{4}$ Majority of these cases are caused as a result of road traffic injuries subsequently by falls and violence.5The prevalence of the road traffic accidents and injuries potentiates a significant load on the mortality as it tends to be one of the ten most leading causes of death worldwide. ${ }^{5,6}$
The cases of traumatic brain injury in the clinical settings are identified majorly with skull fracture, intracranial injury, optic nerve damage, shaken baby syndrome or unspecified head injury. ${ }^{7}{ }^{8}$ As per the "Guidelines for the Management of Traumatic Brain Injury", the surgical evacuation is implemented when the Extradural Hematoma (EDH) is presented with more than 30 milliliters of volume despite of the Glasgow Coma Scale (GCS). The criteria is more specified for the condition when EDH exhibits 15 millimeters of thickness or more along with a midline shift of beyond 5 millimeters. Patients with such indications experience a worse condition of unconsciousness and display lateralizing signs, thus require immediate surgical intervention. ${ }^{9,} 10,11,12,13$

This case study has focused on recent findings in the anesthetic management of patients undergoing craniotomy while awake, and proposed a structured approach 
to the clinical practice of 'local anesthesia' for awake neurosurgery. The case holds a significant character as craniotomy for extradural hematoma is a common procedure under general anesthesia but for extradural hematoma craniotomy is not yet reported under local anesthesia.

\section{Case Presentation}

The situation describes the case of a 40 years old male presented to emergency department of a clinical setting in Hyderabad, Pakistan. The patient has been presented with the loss of consciousness and vomiting since two hours after road traffic accident without any history of comorbidities. Examination revealed dilation of $6 \mathrm{~mm}$ in right pupil with slight reactivity while left pupil displayed reactivity but with constriction. Glasgow Coma Scale scored 8 at the time of presentation with pulse rate of 56 per minute.

The patient has been suspected to have intracranial hematoma for which computed tomography scan (CT scan) was performed that revealed right parieto-occipital extradural hematoma. Following in the standard protocol and in response to the diagnosis, an emergency surgery with general anesthesia had been planned. However, due to the unavailability of the anesthetist due to a long run commitment in an ongoing emergency procedure the intervention was postponed initially. Nonetheless, within 30 minutes of wait the GCS of the patient turned to 4/15 that raised the necessity of an immediate surgical interference. Hence Burr hole craniotomy for the evacuation of extradural hematoma under local anesthesia was performed in order to save the life of the subject as the single burr hole drainage has been presumed to be an insufficient option.
Soon after the admission to the operation room, the patient had been provided with 2\% Xylocaine (lidocaine) plus Adrenaline which was applied to the cutaneous and subcutaneous tissues, respectively. In addition to that, injection of Nalbuphine, 1 ampoule $(10 \mathrm{mg})$, mixed with 1 liter of Normal Saline was continuously administered to the patient during the whole procedure while before and after the practice, intravenous administration of $1 \mathrm{gm}$ Provas has also been carried on the opposite sides.

The muscular layer had been cauterized after which the periosteum has been retracted and four burr hole craniotomy has been made to aid the evacuation of extradural hematoma. The wound has been sealed in layers by applying the tack up sutures and placing the bone flap back. Immediate post-operative evaluation indicated that the GCS had been restored to 8/15. Patient was admitted to intensive care unit for two days in order to keep a follow up and for strict post-surgical management. Repeated CT scan displayed complete clearing of extradural hematoma with underlying small occipital infarction. The patient has been discharged on day 7 of the treatment, without any noted deficits.

\section{Discussion}

Surgical evacuation of the traumatic extradural hematoma is one of the most rewarding surgical procedures in terms of quality of life and years preserved if done without delaying to decrease worst prognosis\& mortality rates. ${ }^{14,15}$ A study conducted in 2013 has stated that subjects presented with extradural hematoma predominantly indicates death with second majority of those who survive but with unfavorable consequences. A very small number is observed to live with favorable outcomes while only few continue to endure 
life completely. ${ }^{16}$ Regardless of timely identification of extradural hematoma, it has been observed in several studies that the fatalities tend to dominate in traumatic brain injuries, particularly in children though the results are seen to be similar in all age groups. ${ }^{17,18}$

Such estimations of the death toll with this present case study, highlights the significance of local anesthesia for extradural hematoma surgery at the time of need.

To add on knowledge, this is the first case to be reported describing the complete evacuation of extradural hematoma using craniotomy under local anesthesia. This procedure can be considered as an alternative to extradural hematoma evacuation in general anesthesia or situations where general anesthesia is not feasible or delayed either due to unavailability of anesthetist or any other reason.

Traumatic brain injury presented with extradural hematoma is taken into account as a neurosurgical emergency that puts up the surgical intervention as the standard procedure. Studies have regarded again that despite being a fatal condition, timely diagnosis and treatment interference in these cases can lower the risk of mortality and produce better results for the patients. ${ }^{19,20}$ We can conclude that the prompt decision of employing the alternative of local anesthesia in this case has been an admirable step taken by the practitioners in the situation that turned out to be successfully beneficial for the patient and saved the precious life, preventing the condition from further preceding as a lethal factor.
Figure 1: Preoperative Scan

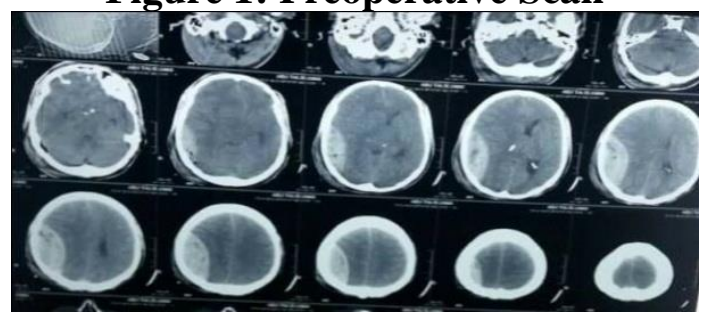

Figure2: Intraoperative After Removal Of Edh

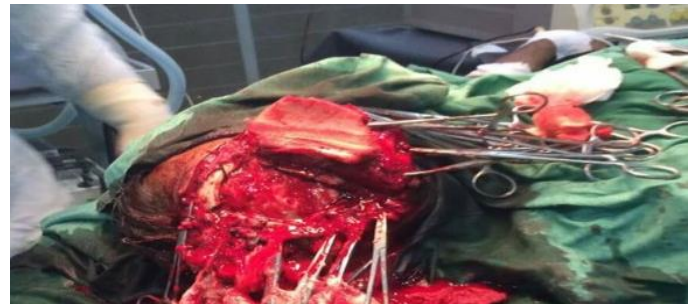

Figure3: Post-Operative Scan

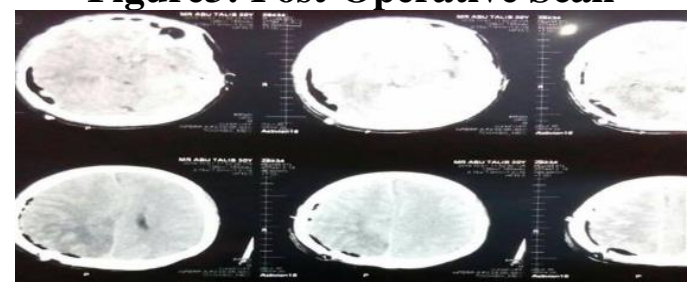

\section{Recommendation}

This procedure can be adopted in emergent cases in situations where general anesthesia is not be feasible or in high risk patients , but caution should be made in selection of patients. We chose middle aged person and would not recommend to proceed this procedure in extremes of age or if patients become painful, the procedure should be abandoned for a while to avoid potential complications like vasovagal shock, Administration of nalbuphine should be made cautiously because of its potential side effect of raising intracranial pressure. We recommend its use as diluted infusion and it should not be used 3-6 hours prior to surgery (peak plasma concentration and half-life), as after craniotomy increased intracranial pressure is manageable. Furthermore, trials should be carried to evaluate the procedure's more potential benefits and side effects, for benefit of patients. 


\section{Conflicts of interest}

None

\section{Acknowledgement}

I would like to thanks Professor Kamran Hussain. Chairman of Neurosurgery Department Sheikh Zayed Hospital Lahore for his broad vision, sincerity towards patients and love towards juniors.

\section{References}

1. World Health Organization. Projections of Mortality and Burden of Disease to 2030: Deaths by Income Group. Geneva; 2002.

2. Marr AL, Coronado VG, eds. Central nervous system injury surveillance data submission standards - 2002. Atlanta, GA: US Department of Health and Human Services, CDC; 2004.

3. Coronado VG, McGuire LC, Sarmiento K, et al. Trends in traumatic brain injury in the U.S. and the public health response: 1995-2009. J Safety Res 2012; 43:229-307.

4. CDC. Report to Congress on mild traumatic brain injury in the United States: steps to prevent a serious public health problem. Atlanta, GA: US Department of Health and Human Services. CDC; 2003.

5. Puvanachandra, P., \&Hyder, A. A. (2009). The burden of traumatic brain injury in Asia: a call for research. Pak J NeurolSci, 4(1), 27-32.

6. Gururaj G. Injuries in India: A National Perspective Burden of disease in India. India; 2005.

7. Badjatia, N., Carney, N., Crocco, T. J., Fallat, M. E., Hennes, H., Jagoda, A. S., \& Pons, P. T. (2008). Guidelines for prehospital management of traumatic brain injury 2nd edition. Prehospital emergency care, 12(SUPPL. 1).

8. Langlois, J. A., Rutland-Brown, W., \& Wald, M. M. (2006). The epidemiology and impact of traumatic brain injury: a brief overview. The Journal of head trauma rehabilitation, 21(5), 375-378.

9. Shen J, Pan JW, Fan ZX, Zhou YQ, Chen Z, Zhan RY: Surgery for contralateral acute epidural hematoma following acute subdural hematoma evacuation: five new cases and a short literature review. ActaNeurochir (Wien). 2013, 155 (2): 335-341.
10. Le TH, Gean AD: Neuroimaging of traumatic brain injury. Mt Sinai J Med. 2009, 76 (2): 145162. 10.1002/msj.20102.

11. Zammit C, Knight WA: Severe traumatic brain injury in adults. Emerg Med Pract. 2013, 15 (3): 1-28. Epub 2013 Feb 20.

12. Udoh DO: Bilateral post-traumatic acute extradural hematomas: a report of four cases and review of literature. Niger J ClinPract. 2012, 15 (1): 104-107. 10.4103/11193077.94111 .

13. Bilotta, Federico, and Giovanni Rosa. "'Anesthesia'for awake neurosurgery." Current Opinion in Anesthesiology 22.5 (2009): 560565.

14. Su, T. M., Lee, T. H., Chen, W. F., Lee, T. C., \& Cheng, C. H. (2008). Contralateral acute epidural hematoma after decompressive surgery of acute subdural hematoma: clinical features and outcome. Journal of Trauma and Acute Care Surgery, 65(6), 1298-1302.

15. Cuenca, P. J., Tulley, E. B., Devita, D., \& Stone, A. (2004). Delayed traumatic spinal epidural hematoma with spontaneous resolution of symptoms. The Journal of emergency medicine, 27(1), 37-41.

16. Leitgeb, J., Mauritz, W., Brazinova, A., Majdan, M., \&Wilbacher, I. (2013). Outcome after severe brain trauma associated with epidural hematoma. Archives of orthopaedic and trauma surgery, 133(2), 199-207.

17. Gerlach, R., Dittrich, S., Schneider, W., Ackermann, H., Seifert, V., \&Kieslich, M. (2009). Traumatic epidural hematomas in children and adolescents: outcome analysis in 39 consecutive unselected cases. Pediatric emergency care, 25(3), 164-169.

18. Jung, S. W., \& Kim, D. W. (2012). Our experience with surgically treated epidural hematomas in children. Journal of Korean Neurosurgical Society, 51(4), 215-218.

19. Cheung, P. S., Lam, J. M., Yeung, J. H., Graham, C. A., \& Rainer, T. H. (2007). Outcome of traumatic extradural haematoma in Hong Kong. Injury, 38(1), 76-80.

20. Rocchi, G., Caroli, E., Raco, A., Salvati, M., \&Delfini, R. (2005). Traumatic epidural hematoma in children. Journal of child neurology, 20(7), 56 